Statistical model for prebreakdown current jumps and breakdown caused by single traps in magnetic tunnel junctions

J. Das, R. Degraeve, G. Groeseneken, S. Stein, H. Kohlstedt, G. Borghs, and J. De Boeck

Citation: Journal of Applied Physics 94, 2749 (2003);

View online: https://doi.org/10.1063/1.1592300

View Table of Contents: http://aip.scitation.org/toc/jap/94/4

Published by the American Institute of Physics

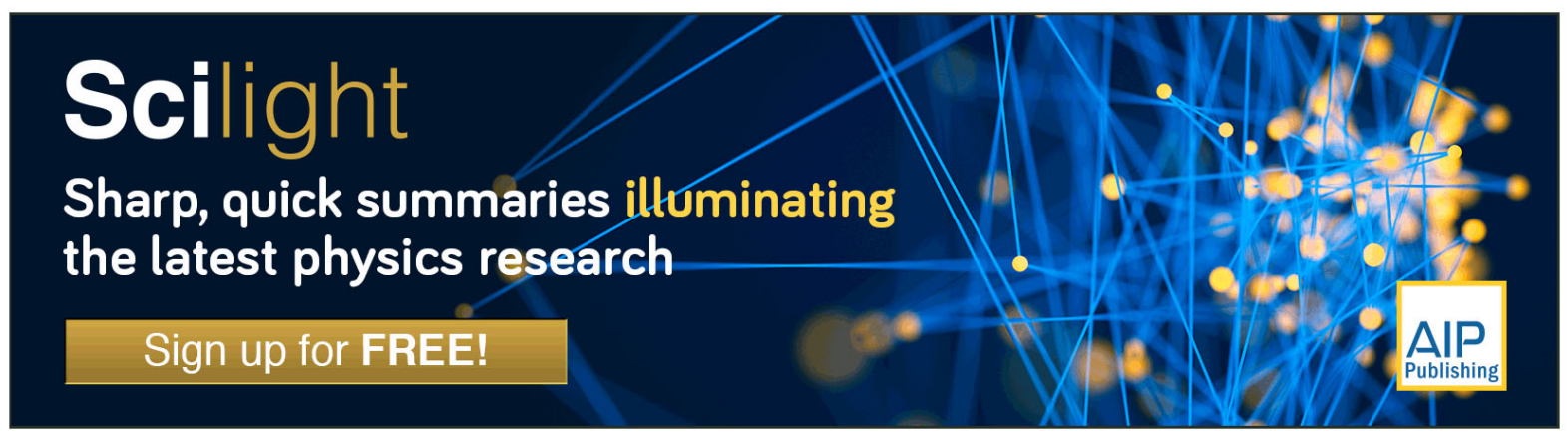




\title{
Statistical model for prebreakdown current jumps and breakdown caused by single traps in magnetic tunnel junctions
}

\author{
J. Das, ${ }^{\text {a),b) }}$ R. Degraeve, and G. Groeseneken ${ }^{\text {b) }}$ \\ Imec, Kapeldreef 75, B-3001 Leuven, Belgium \\ S. Stein and $\mathrm{H}$. Kohlstedt \\ Institut für Festkörperforschung, Forschungszentrum Jülich GmbH, 52425 Jülich, Germany
}

G. Borghs and J. De Boeck

Imec, Kapeldreef 75, B-3001 Leuven, Belgium

(Received 7 October 2002; accepted 23 May 2003)

To obtain reliable magnetic tunnel junctions (MTJs) for sensor and memory applications, the quality of the $\mathrm{Al}_{2} \mathrm{O}_{3}$ tunnel barrier is extremely important. Here, we studied the reliability of MTJs with a $1.6 \mathrm{~nm} \mathrm{Al} \mathrm{Al}_{3}$ tunnel barrier formed by ultraviolet light assisted oxidation. In the stress measurements, prebreakdown current jumps and, finally, breakdown are observed. We show, by using statistics, that both the current jumps and the final breakdown can be attributed to single trap generation. Moreover, we can relate the current jump height to the trap location. In this way, we reveal the breakdown mechanism in MTJs and illustrate the importance of reliability studies.

(C) 2003 American Institute of Physics. [DOI: 10.1063/1.1592300]

The integration of magnetic tunnel junctions (MTJs) into magnetic random access memories (MRAM) will rely on the excellent magnetic and electrical properties of the MTJ. Surprisingly, only few reports on the reliability of MTJs are available. In most present MTJ reliability studies, ${ }^{1-4}$ ramp tests were performed on the MTJs. However, in addition, constant stress, tests are better suited to get an insight in the breakdown mechanisms. Furthermore, since different oxidation methods are used to make the $(\sim 1 \mathrm{~nm}) \mathrm{Al}_{2} \mathrm{O}_{3}$ tunnel barrier in MTJs, we believe that reliability studies are important to investigate $\mathrm{Al}_{2} \mathrm{O}_{3}$ quality. Recently, preliminary constant voltage tests were performed on naturally oxidized MTJs, ${ }^{5}$ concluding that in this case, breakdown is initiated by extrinsic defects. Here, we will demonstrate that intrinsic breakdown behavior can be obtained by using a different oxidation technique.

We present an in-depth analysis of the degradation and breakdown of MTJs with a $1.6 \mathrm{~nm} \mathrm{Al}_{2} \mathrm{O}_{3}$ tunnel barrier formed by ultraviolet (UV) light-assisted oxidation. We will demonstrate that the reliability concepts used for $\mathrm{SiO}_{2}$ can also be applied to $\mathrm{Al}_{2} \mathrm{O}_{3}$. We will show that the observed degradation is due to the generation of single traps. Finally, we can link the generation of traps, the prebreakdown current through these traps, and the breakdown of MTJs. This study illustrates the fact that breakdown tests can lead to a quality test of the available oxidation methods for MTJs.

Contrary to the $\mathrm{Al}_{2} \mathrm{O}_{3}$ tunnel barrier in MTJs, the reliability of $\mathrm{SiO}_{2}$ capacitors is a well-established research topic. It is generally accepted that intrinsic breakdown of thin $\mathrm{SiO}_{2}$ layers is related to the generation of a percolation path of traps in the oxide. ${ }^{6}$ Recently, evidence has been presented that this mechanism also causes breakdown in thick $\mathrm{Al}_{2} \mathrm{O}_{3}$

\footnotetext{
${ }^{a)}$ Electronic mail: jdas@imec.be

${ }^{b}$ Also at: Electronic Engineering Department, KULeuven, Belgium.
}

layers. ${ }^{7}$ Applying an electrical stress to the oxide leads to trap generation. At a certain moment, a conduction path of neighboring traps is formed between the two interfaces and, as a result, the oxide breaks down. When scaling down the oxide thickness, fewer traps are needed to form this breakdown path. In the limit, for very thin oxides, only one wellpositioned trap is sufficient to trigger a breakdown event. Recently, prebreakdown current steps were observed in stress measurements on small $\left(2 \mu \mathrm{m}^{2}\right) \mathrm{SiO}_{2}$ capacitors. These are attributed to the creation of two-trap conduction paths, which are physically identical to breakdown paths except that their conductivity is insufficient to cause the electrical runaway effects that accompany breakdown. ${ }^{8}$

The detailed layer sequence of the MTJ stack, used in this study, is the following: $\mathrm{Si} / \mathrm{SiO}_{2}$ substrate/Ta $5 \mathrm{~nm} / \mathrm{Ni}_{80} \mathrm{Fe}_{20} 3 \mathrm{~nm} / \mathrm{FeMn} 20 \mathrm{~nm} / \mathrm{Co}_{90} \mathrm{Fe}_{10} 3 \mathrm{~nm} / \mathrm{Al} 1.3 \mathrm{~nm}$ + oxidation $/ \mathrm{Co}_{90} \mathrm{Fe}_{10} 3 \mathrm{~nm} / \mathrm{Ni}_{80} \mathrm{Fe}_{20} 7 \mathrm{~nm} / \mathrm{Cu} 5 \mathrm{~nm}$. All layers are deposited by sputtering. To obtain the $\mathrm{Al}_{2} \mathrm{O}_{3}$ tunnel barrier, the deposited $\mathrm{Al}$ layer was exposed to an atmosphere of $5 \mathrm{mbar} \mathrm{O}_{2}$ and UV light. ${ }^{9}$ MTJs with different top electrode sizes were fabricated. First, the bottom and top electrode are defined by a two-step ion milling. This is followed by a self-aligned $\mathrm{SiO}_{2}$ passivation step. After a final metallization deposition, which provides contacts to the top and bottom electrodes, an anneal was performed at $250{ }^{\circ} \mathrm{C}$ in a magnetic field. The MTJs showed a tunnelmagnetoresistance signal of $30 \%$.

A constant voltage stress at different voltages was applied to the MTJs until breakdown occurred. Figure 1 shows a typical current versus time trace of a stress measurement on a $12.5 \mu \mathrm{m}^{2}$ MTJ at $1.1 \mathrm{~V}$. The time-to-breakdown $\left(t_{\mathrm{BD}}\right)$ is a statistically distributed parameter. The cumulative time-tobreakdown distributions $F\left(t_{\mathrm{BD}}\right)$ (at stress levels from $1.05 \mathrm{~V}$ up to $1.125 \mathrm{~V}$ ) are fitted with a Weibull function having two parameters: The distribution slope $\beta$ and the $63 \%$ value $t_{63}$. 


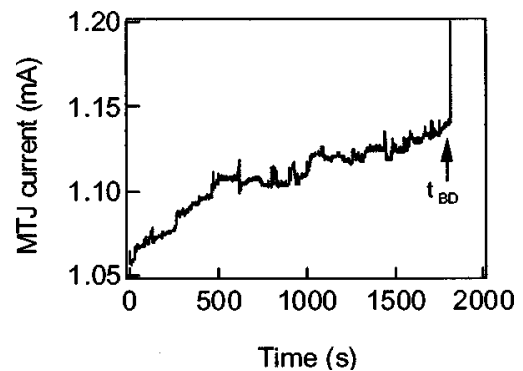

FIG. 1. Typical stress curve $(V=1.1 \mathrm{~V})$ of a $12.5 \mu \mathrm{m}^{2}$ MTJ. Several prebreakdown current jumps are observed before the MTJ finally breaks down at $t=t_{\mathrm{BD}}$.

The measured $t_{\mathrm{BD}}$ distributions, together with the Weibull fits, are shown in Fig. 2. As explained in our previous study, ${ }^{5}$ it was necessary to verify the scaling of $t_{\mathrm{BD}}$ with the area of the devices. It was found that $t_{\mathrm{BD}}$ scales properly with the area, so breakdown due to perimeter effects can be excluded.

From Fig. 1, it is clear that several sudden current jumps are already observed before the final MTJ breakdown occurs. These current jumps are similar to the prebreakdown events in thin $\mathrm{SiO}_{2},{ }^{8}$ and can be further analyzed to provide information on the defect position. It is noteworthy that this defect or trap creates a metastable state in the oxide. Due to the limited oxide thickness, a trapped electron will not remain in this state very long but it will tunnel out rapidly toward the cathode. As a result, traps give rise to additional conduction paths.

For each measurement (at $V=1.1 \mathrm{~V}$ ), the time $t_{i}$, which corresponds to the first current step larger than $\Delta I_{i}$, was extracted. In this way, for each $\Delta I_{i}$, a distribution of $t_{i}$ was obtained (Fig. 3). All of the distributions were fitted with a maximum likelihood algorithm. The Weibull slope remains constant for all distributions and equals 0.8. We will now develop a model that relates the observed current jumps and breakdown of the MTJ to the generation of traps in $\mathrm{Al}_{2} \mathrm{O}_{3}$. Recently, for $2.4 \mathrm{~nm} \mathrm{SiO}{ }_{2}$ capacitors, it was shown that $t_{i}$ (time to observe a current jump $>\Delta I_{i}$ ), is Weibull distributed and the Weibull slope $\beta$ decreases for decreasing $\Delta I_{i} .{ }^{8}$ These observations were statistically explained with a twotrap percolation model. For ultrathin oxide layers, however,

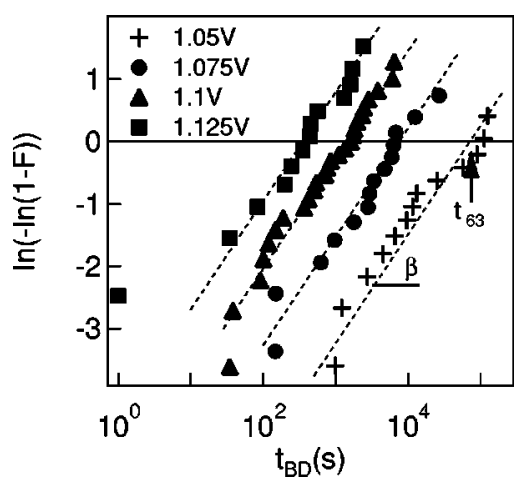

FIG. 2. The distributions of time-to-breakdown $\left(t_{\mathrm{BD}}\right)$ at different stress levels. The cumulative distributions $F$ are plotted on a Weibull scale: This results in straight lines with slope $\beta$. The $63 \%$ values of $t_{\mathrm{BD}}\left(=t_{63}\right)$ are located where the distributions intersect with the zero axis.

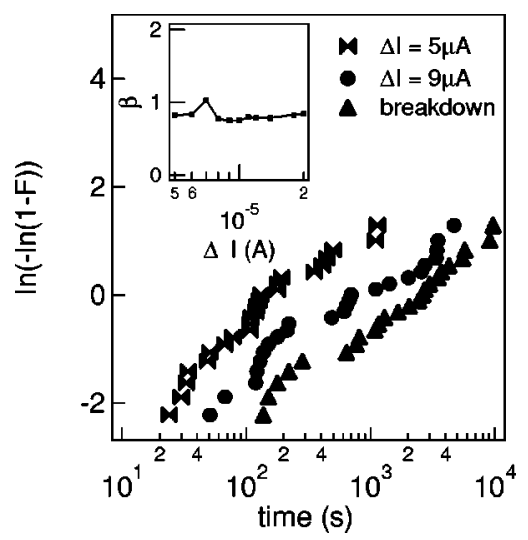

FIG. 3. Distributions of the time $t_{i}$ to observe the first current step higher than a given current value $\Delta I_{i}$. As this current gets higher, the Weibull plot shifts to the right-hand side. The right-hand side curve is the breakdown distribution at $1.1 \mathrm{~V}$. The inset shows the Weibull slope $\beta$ which remains constant.

we expect both the prebreakdown and the breakdown to be triggered by single traps created during the stress. To prove this, we start from a general relation for trap generation similar to that in $\mathrm{SiO}_{2}:^{8}$

$$
D_{\mathrm{ot}}=C t^{m},
$$

with $D_{\text {ot }}$ as the trap density, $C$ as a constant, $t$ as the stress time, and $m$ as the (logarithmic) trap generation rate. For events corresponding to current jumps $>\Delta I_{i}$, a certain trap density $D_{\mathrm{ot}, i}$ is required. For all single trap events $\Delta I_{i}, D_{\mathrm{ot}, i}$ is Poisson distributed ( $=$ Weibull distributed with slope $\beta$ $=1)$. Poisson distributions for $D_{\text {ot }, i}$ can be transformed into Weibull distributions for $t_{i}$ using Eq. (1). The Weibull slope for these $t_{i}$ distributions becomes $\beta=m$, a constant for all $\Delta I_{i}$. This constant slope for the $t_{i}$ distributions is, hence, a characteristic of the single trap generation and breakdown. This is in contrast with the varying slope observed for multiple trap generation. ${ }^{8}$ Coming back to our experiments, the measured $\beta$ values are constant for all $\Delta I_{i}$ (Fig. 3). Consequently, we can conclude that all the different prebreakdown current jumps, as well as the final breakdown, are intrinsic degradation effects caused by single trap generation. Note that a $\beta$ value $<1$ is usually attributed to extrinsic breakdown mechanisms. However, in this case, we have a generation of single traps, which is an intrinsic degradation mechanism. Using the transformation of Eq. (1), where the trap generation rate $m=0.8$, we clearly see that the creation of single traps leads to a $t_{i}$ distribution with Weibull slope $\beta$ $=m=0.8$.

We will now derive a relation between the measured current step magnitudes $\Delta I_{i}$ and the position of the traps in the oxide. To achieve this, the percolation concept, as it has been applied for thin $\mathrm{SiO}_{2}$, will be extended to model single trap conduction. The current through each conduction path $\Delta I$ is determined by the longest trap-to-interface distance $x_{\text {perc }}$ as defined in Fig. 4 . The probability $P\left(N, x_{\text {perc }}\right)$ to have a device with exactly $N$ traps (at a density of traps $D_{\text {ot }}$ ) and at least one single trap percolation path with $x<x_{\text {perc }}$, is given by 


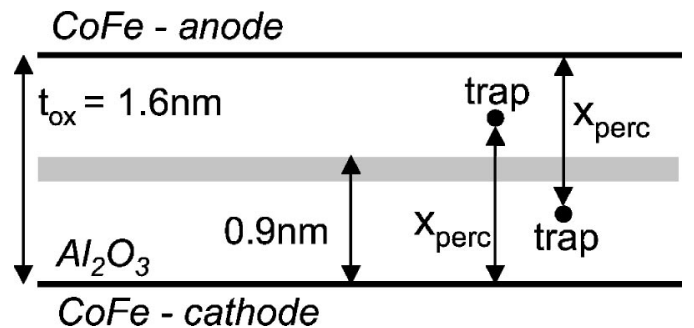

FIG. 4. $x_{\text {perc }}$ is defined as the longest distance from the trap to one of both interfaces. If a trap is positioned within the gray part of the tunnel barrier $\left(x_{\text {perc }}<0.9 \mathrm{~nm}\right)$, breakdown will occur.

$P\left[N, x_{\mathrm{perc}}\right]=\left[1-\left(2-\frac{2 x_{\mathrm{perc}}}{t_{\mathrm{ox}}}\right)^{N}\right] \cdot\left[\frac{\left(A t_{\mathrm{ox}} D_{\mathrm{ot}}\right)^{N}}{N !} e^{-A t_{\mathrm{ox}} D_{\mathrm{ot}}}\right]$,

with $t_{\mathrm{ox}}$ is the oxide thickness and $A$ is the oxide area. Note that the first factor of Eq. (2) gives the probability to have at least one percolation path with $x<x_{\text {perc }}$ in an oxide with exactly $N$ defects and the second factor gives the probability to have exactly $N$ defects. From Eq. (2), we can easily derive the probability to have at least one percolation path with $x$ $<x_{\text {perc }}$, by summing Eq. (2) for $N: 1 \rightarrow \infty$ (at fixed $D_{\text {ot }}$ ). Setting this probability to 0.63 , gives the relation between $x_{\text {perc }}$ and $D_{\mathrm{ot}, 63}$ :

$$
\frac{1}{A t_{\mathrm{ox}} D_{\mathrm{ot}, 63}}=\frac{2 x_{\mathrm{perc}}}{t_{\mathrm{ox}}}-1 .
$$

This relation gives the average $D_{\text {ot }}$ needed to create at least one trap at $x<x_{\text {perc }}$.

Combining Eqs. (1) and (3), $x_{\text {perc }}$ can be related to the $t_{i}$ distribution shown in Fig. 3(a). The constant $C$ in Eq. (1) was calculated (similar as for $\mathrm{SiO}_{2}$ capacitors) ${ }^{8}$ from the assumption that breakdown occurs when a trap with $x_{\text {perc }}<0.9 \mathrm{~nm}$ is created. Taking this into account, we can finally construct the current through the traps $\left(I_{\text {trap }}=\Delta I\right)$ versus $x_{\text {perc }}$ (Fig. 5). These results are very useful to reveal the physical conduction mechanism through traps in MTJs, although more inves-

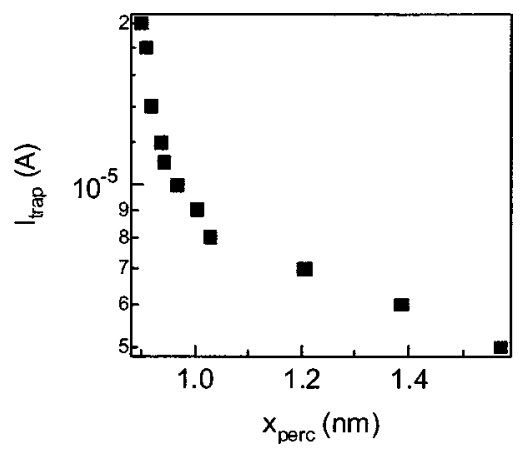

FIG. 5. Relation between the current through the traps $\left(I_{\text {trap }}\right)$ and the trap position within the barrier $\left(x_{\text {perc }}\right)$ at stress voltage $\mathrm{V}=1.1 \mathrm{~V}$. tigation will be required. We attempted to model this curve by a trap-assisted tunneling model, ${ }^{10}$ but unrealistically high trap cross sections were found $\left(>10^{-13} \mathrm{~cm}^{2}\right)$. This proves that at the position of the trap, the tunneling barrier is distorted and the insulating properties are severely affected. We assume that the creation of traps will locally lower the barrier height, which results in a much higher local conductivity. To get additional insight in the trap creation processes, other techniques, like e.g., ballistic electron emission microscopy ${ }^{11}$ and noise characterization, ${ }^{12,13}$ can be useful, in addition to the electrical stress measurements which give direct quantitative information on MTJ devices.

In summary, we analyzed in detail the reliability of 1.6 nm UV-oxidized $\mathrm{Al}_{2} \mathrm{O}_{3}$ MTJs. Intrinsic prebreakdown and breakdown were observed after constant voltage stress. These events are caused by the creation of single trap conduction paths and the relation of current versus trap position has been determined applying a percolation concept. Besides the eventual breakdown, the prebreakdown generation of leakage paths will result in a resistance drift of the MTJ and this may jeopardize the MRAM function. This should be taken into account in a reliability prediction. Finally, this study should be extended toward other oxidation techniques (and different oxide thicknesses) to obtain a global quantitative comparison between the available oxidation methods.

One of the authors (J.D.) acknowledges the IWT for financial funding. E. Vandenplas is acknowledged for processing assistance. This work is partly supported by the HGFStrategiefonds "Magnetoelectronic" and the BMBFLeitprojekt: "Magnetoelektronik."

${ }^{1}$ W. Oepts, H. J. Verhagen, W. J. M. de Jonge, and R. Coehoorn, Appl. Phys. Lett. 73, 2363 (1998).

${ }^{2}$ K. Shimazawa, N. Kasahara, J. J. Sun, A. Araki, H. Morita, and M. Matsuzaki, J. Appl. Phys. 87, 5194 (2000).

${ }^{3}$ J. Schmalhorst, H. Brückl, M. Justus, A. Thomas, G. Reiss, M. Vieth, G. Gieres, and J. Wecker, J. Appl. Phys. 89, 586 (2001).

${ }^{4}$ B. Oliver, Q. He, X. Tang, and J. Nowak, J. Appl. Phys. 91, 4348 (2002).

${ }^{5}$ J. Das, R. Degraeve, P. Roussel, G. Groeseneken, G. Borghs, and J. De Boeck, J. Appl. Phys. 97, 7712 (2002).

${ }^{6}$ R. Degraeve, J. L. Ogier, R. Bellens, P. J. Roussel, G. Groeseneken, and H. E. Maes, IEEE Trans. Electron Devices 45, 904 (1998).

${ }^{7}$ A. Kerber, E. Cartier, R. Degraeve, L. Pantisano, P. Roussel, and G. Groeseneken, VLSI Technology Symp. Dig. of Techn. Papers, (2002), pp. $76-77$.

${ }^{8}$ R. Degraeve, B. Kaczer, F. Schuler, M. Lorenzini, D. Wellekens, P. Hendrickx, J. Van Houdt, L. Haspeslagh, G. Tempel, and G. Groeseneken, Tech. Dig. - Int. Electron Devices Meet. 2001, 6.2.1 (2001).

${ }^{9}$ P. Rottländer, H. Kohlstedt, P. Grünberg, and E. Girgis, J. Appl. Phys. 87, 6067 (2000)

${ }^{10}$ F. Schuler, R. Degraeve, P. Hendrickx, and D. Wellekens, Int. Reliability Physics Symp. (2002), pp. 26-33.

${ }^{11}$ W. H. Rippard, A. C. Perrella, and R. A. Buhrman, Appl. Phys. Lett. 78, 1601 (2001).

${ }^{12}$ C. T. Rogers and R. A. Buhrman, Phys. Rev. Lett. 55, 859 (1985).

${ }^{13}$ F. Crupi, B. Neri, and S. Lombardo, IEEE Electron Device Lett. 32, 319 (2000) 